Cite this: Nanoscale, 2014, 6, 4566

Received 12th February 2014 Accepted 2nd March 2014

DOI: $10.1039 / c 4 n r 00783 b$

www.rsc.org/nanoscale

\section{van der Waals trilayers and superlattices: modification of electronic structures of $\mathrm{MoS}_{2}$ by intercalation $\uparrow$}

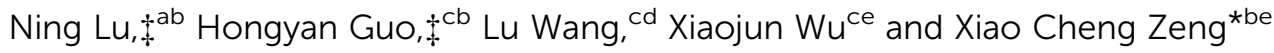

\begin{abstract}
We perform a comprehensive first-principles study of the electronic properties of van der Waals (vdW) trilayers via intercalating a twodimensional (2D) monolayer ( $\mathrm{ML}=\mathrm{BN}, \mathrm{MoSe}_{2}, \mathrm{WS}_{2}$, or WSe $\mathrm{We}_{2}$ ) between a $\mathrm{MoS}_{2}$ bilayer to form various $\mathrm{MoS}_{2} / \mathrm{ML} / \mathrm{MoS}_{2}$ sandwich trilayers. We find that the $\mathrm{BN}$ monolayer is the most effective sheet to decouple the interlayer vdW coupling of the $\mathrm{MoS}_{2}$ bilayer, and the resulting sandwich trilayer can recover the electronic structures of the $\mathrm{MoS}_{2}$ monolayer, particularly the direct-gap character. Further study of the $\mathrm{MoS}_{2}$ /BN superlattices confirms the effectiveness of the BN monolayer for the decoupling of the $\mathrm{MoS}_{2}-\mathrm{MoS}_{2}$ interaction. In addition, the intercalation of a transition-metal dichalcogenide (TMDC) $\mathrm{MoSe}_{2}$ or $\mathrm{WSe}_{2}$ sheet makes the sandwich trilayer undergo an indirect-gap to direct-gap transition due to the newly formed heterogeneous $\mathrm{S} / \mathrm{Se}$ interfaces. In contrast, the $\mathrm{MoS}_{2} / \mathrm{WS}_{2} / \mathrm{MoS}_{2}$ sandwich trilayer still retains the indirect-gap character of the $\mathrm{MoS}_{2}$ bilayer due to the lack of the heterogeneous $\mathrm{S} / \mathrm{Se}$ interfaces. Moreover, the 3D superlattice of the $\mathrm{MoS}_{2} / \mathrm{TMDC}$ heterostructures also exhibits similar electronic band characters to the $\mathrm{MOS}_{2} / \mathrm{TMDC} / \mathrm{MoS}_{2}$ trilayer heterostructures, albeit a slight decrease of the bandgap compared to the trilayers. Compared to the bulk $\mathrm{MoS}_{2}$, the 3D MoS$/$ /TMDC superlattice can give rise to new and distinctive properties. Our study offers not only new insights into electronic properties of the vdW multilayer heterostructures but also guidance in designing new heterostructures to modify electronic structures of 2D TMDC crystals.
\end{abstract}

\footnotetext{
${ }^{a}$ Center for Nano Science and Technology, Department of Physics, Anhui Normal University, Wuhu, Anhui, 241000, China

${ }^{b}$ Department of Chemistry and Department Mechanics and Materials Engineering, University of Nebraska-Lincoln, Lincoln, NE 68588, USA. E-mail: xzeng1@unl.edu

${ }^{c}$ CAS Key Lab of Materials for Energy Conversion, Department of Materials Science and Engineering, University of Science and Technology of China, Hefei, Anhui 230026, China

${ }^{d}$ Department of Physics, University of Nebraska-Omaha, Omaha, NE 68182, USA ${ }^{e}$ Hefei National Laboratory for Physical Sciences at the Microscale, University of Science and Technology of China, Hefei, Anhui 230026, China

$\dagger$ Electronic supplementary information (ESI) available. See DOI: $10.1039 / \mathrm{c} 4 \mathrm{nr} 00783 \mathrm{~b}$

$\ddagger$ Both authors contributed equally to this work
}

\section{Introduction}

Many experiments have demonstrated that two-dimensional (2D) transition-metal dichalcogenides (TMDCs) such as 2D $\mathrm{MoS}_{2}$ and $\mathrm{WS}_{2}$ crystals possess novel electronic, ${ }^{1-4}$ optical, ${ }^{5-8}$ catalytic, ${ }^{9,10}$ and mechanical properties. ${ }^{11-14}$ For example, electronic properties of $2 \mathrm{D} \mathrm{MoS}$ crystals can be sensitive to the number of layers, that is, the $\mathrm{MoS}_{2}$ monolayer exhibits a direct bandgap with a value of $\sim 1.8 \mathrm{eV}$ while a bilayer $\mathrm{MoS}_{2}$ exhibits an indirect bandgap with a value of $\sim 1.5 \mathrm{eV} .{ }^{15}$ As a result, significant enhancement of photoluminescence has been observed when $\mathrm{MoS}_{2}$ is thinned to a single layer. ${ }^{8,14}$ Previous study has also shown that when the $\mathrm{MoS}_{2}$ bilayer is pulled apart into two separated monolayers, the direct transition $(K-K)$ is insensitive to the separation while the indirect transition $(I-K)$ increases dramatically. ${ }^{16}$ It seems that the distance between the two monolayers or the interlayer vdW interaction can notably affect the electronic structures of two-dimensional (2D) layered TMDCs. Thus, one may ask two closely related questions: "can the $\mathrm{MoS}_{2}$ bilayer be effectively decoupled via intercalation of a 2D sheet without being pulled far too apart?" or "To what extent, can the intercalation of a 2D sheet affect electronic properties of the $\mathrm{MoS}_{2}$ bilayer?" The intercalation of a 2D sheet into the $\mathrm{MoS}_{2}$ bilayer gives rise to a hybrid trilayer, coined as the $\mathrm{vdW}$ heterostructures by Geim and Grigorieva. ${ }^{17}$ Recently, successful fabrication of multilayer vdW heterostructures by stacking one layer on top of another in a precisely controlled sequence has been demonstrated experimentally. ${ }^{18-20}$ The artificial vdW heterostructures can exhibit new and unusual properties that differ from their own constituent layers. For example, the vertical field-effect transistor and memory cell made of TMDC/graphene heterostructures ${ }^{19,21-23}$ as well as layered hybrids of $\mathrm{MoS}_{2}$ and $\mathrm{WS}_{2}$ have been realized in the laboratory. ${ }^{24}$ Previous theoretical studies suggest that the direct-gap character of the $\mathrm{MoS}_{2}$ monolayer can be retained in certain $\mathrm{MoS}_{2}$ heterobilayer structures whose electronic properties can be further tuned by an in-plane strain or a vertical electric field. ${ }^{25-29}$ In addition, the insulating $\mathrm{BN}$ monolayer is a good substrate for 
protecting high quality graphene electronics. ${ }^{30}$ A type-I band alignment for the $\mathrm{BN}$ monolayer and $\mathrm{MoS}_{2}$ monolayer is also reported..$^{25} 3 \mathrm{D}$ heterostructures such as superlattices are predicted to possess new properties that differ from their corresponding bulk structures, thereby opening a new way of materials design. ${ }^{31}$

The focus of this study is to investigate effects of intercalation of either an insulating $\mathrm{BN}$ monolayer or a semiconducting TMDC monolayer $\left(\mathrm{MoSe}_{2}, \mathrm{WS}_{2}\right.$, or $\left.\mathrm{WSe}_{2}\right)$ into a $\mathrm{MoS}_{2}$ bilayer on the electronic properties of the vdW trilayer heterostructure and the corresponding vdW superlattice. Our computational results suggest that the $\mathrm{BN}$ monolayer is an ideal sheet to decouple the $\mathrm{MoS}_{2}$ bilayer while a $\mathrm{MoSe}_{2}$ or $\mathrm{WSe}_{2}$ sheet can turn the indirectgap of the $\mathrm{MoS}_{2}$ bilayer into a direct-gap trilayer.

\section{Computational methods}

All calculations are performed within the framework of spinpolarized plane-wave density functional theory (PW-DFT), implemented in the Vienna $a b$ initio simulation package (VASP 5.3). ${ }^{32,33}$ The Perdew-Burke-Ernzerhof (PBE) functional and projector augmented wave (PAW) potentials are used. ${ }^{34-36}$ The effect of vdW interaction is accounted for by using the dispersion corrected DFT (optB88-vdW functional). ${ }^{37,38}$ The vacuum length between two adjacent images in the supercell is longer than $15 \AA$. An energy cutoff of $500 \mathrm{eV}$ is adopted for the plane-wave expansion of the electronic wave function. Geometric structures are relaxed until the force on each atom is less than $0.01 \mathrm{eV}^{-1}$ and the convergence criteria for energy is $1 \times 10^{-5} \mathrm{eV}$.

Note that the optimized $\mathrm{MoS}_{2}$ monolayer exhibits a cell parameter of $3.18 \AA$, while the cell parameter of a $h$-BN monolayer is $2.52 \AA$, in good agreement with previous results. ${ }^{39,40}$ As such, the $5 \times 5 \mathrm{BN}$ supercell almost perfectly matches the $4 \times 4$ $\mathrm{MoS}_{2}$ supercell with the lattice mismatch less than 1\%. For the
$\mathrm{MoS}_{2} / \mathrm{BN} / \mathrm{MoS}_{2}$ trilayer, the supercell is fixed while the atomic coordinates are relaxed. For other sandwich systems containing TMDC $\mathrm{MoSe}_{2}, \mathrm{WS}_{2}$, or WSe $\mathrm{W}_{2}$, a $1 \times 1$ cell is used due to the fact that their lattice parameters are close to that of $\mathrm{MoS}_{2}$. In these cases, both the cell length and atomic coordinates are relaxed to obtain the lattice parameters at the lowest total energy. Bader's atom in molecule (AIM) method based on charge density topological analysis is used for computing charge population. ${ }^{\mathbf{4 1}}$ Once the optimized structures are obtained, a hybrid functional in the Heyd-Scuseria-Ernzerhof (HSE06) form is used to give more accurate bandgaps. ${ }^{42}$ The spin-orbit (SO) interaction is included in all the band-structure calculations. ${ }^{43}$

\section{Results and discussion}

\section{Intercalation of a $\mathrm{MoS}_{2}$ bilayer with a $\mathrm{BN}$ monolayer}

First, DFT/PBE calculations show that monolayer $\mathrm{MoS}_{2}$ is a semiconductor with a direct bandgap of $1.60 \mathrm{eV}$ (see Table 1), in agreement with previous calculation. ${ }^{29}$ The $\mathrm{MoS}_{2}$ bilayer with the most stable C7 stacking, however, is a semiconductor with an indirect bandgap of $1.31 \mathrm{eV}$. HSE06 calculation enlarges the bandgaps of monolayer and bilayer $\mathrm{MoS}_{2}$ to 2.06 and $1.81 \mathrm{eV}$, respectively. For the $\mathrm{BN}$ monolayer, $\mathrm{PBE}$ calculation shows it is a semiconductor with a wide bandgap of $4.66 \mathrm{eV}$ (Table 2).

Next, various $\mathrm{MoS}_{2} / \mathrm{BN} / \mathrm{MoS}_{2}$ trilayer systems are built for which the lateral locations of the $\mathrm{MoS}_{2}$ monolayer and $\mathrm{BN}$ monolayer in different supercells are shown in Fig. 1. Specifically, we consider four different stacking orders: A1B1A1, A1B2A1, A1B1A2, and A1B1A3. PBE optimizations show that the total-energy differences among these configurations are typically less than $0.01 \mathrm{eV}$ per formula cell, and the different stacking orders have little effect on the electronic structures. The binding energy of a trilayer, which measures the interlayer vdW interaction per supercell, is defined as: $E_{\mathrm{BE}}=2 E_{\mathrm{MoS}_{2}}+E_{\mathrm{BN}}$ - $E_{\mathrm{MoS}_{2} / \mathrm{BN} \mathrm{MoS}_{2}}$, where $E_{\mathrm{MoS}_{2}}$ is the total energy of a $\mathrm{MoS}_{2}$

Table 1 The distance $d$ (in $\AA$ ) between two nearest-neighbor monolayers as shown in Fig. 1. The binding energy $E_{\mathrm{BE}}$ (in eV) per formula unit. The computed bandgaps $E_{\mathrm{g}}$ (in eV) of $\mathrm{MoS}_{2}$ monolayer $\left(\mathrm{ML}-\mathrm{MoS}\right.$ ), bilayer $\left(\mathrm{BL}-\mathrm{MoS}_{2}\right)$, trilayer $\left(\mathrm{MoS}_{2} / \mathrm{BN} / \mathrm{MoS}_{2}\right)$ heterostructures and related superlattice (SL) with different stacking orders. The SO effect is included in HSE06 calculation of the bandgap

\begin{tabular}{lllllllll}
\hline & ML-MoS & BL-MoS2 & A1B1A1 & A1B2A1 & A1B1A2 & A1B1A3 & SL-A1B1 & SL-A1B2 \\
\hline$d$ & - & 3.09 & 3.36 & 3.36 & 3.36 & 3.36 & 3.35 \\
$E_{\mathrm{BE}}$ & - & 0.22 & 0.36 & 0.36 & 0.37 & 0.36 & 0.38 \\
$E_{\mathrm{g}}$ (PBE) & 1.60 & 1.31 -indirect & 1.58 & 1.58 & 1.58 & 1.58 & 1.69 \\
$E_{\mathrm{g}}$ (HSE06) & 2.06 & 1.81 -indirect & 2.00 & - & - & - & - & -
\end{tabular}

Table 2 Computed PBE $E_{g}$ (PBE) and HSEO6 $E_{g}$ (HSE) bandgaps of $\mathrm{MoS}_{2} / \mathrm{ML} / \mathrm{MoS}_{2}$ trilayers $\left(\mathrm{ML}=\mathrm{MoSe}_{2}, \mathrm{WS}_{2}\right.$ or WSe $)$ in $\mathrm{ABA}$ and $\mathrm{ACA}$ stacking (see Fig. 3). The PBE ( $\left.E_{g}(\mathrm{PBE}) \_S L\right)$ and HSEO6 ( $\left.E_{\mathrm{g}}(\mathrm{HSE}) \_S L\right)$ bandgaps of $\mathrm{MoS}_{2} / \mathrm{ML}$ superlattice $\left(\mathrm{ML}=\mathrm{MoSe}_{2}, \mathrm{WS}_{2}, \mathrm{WSe}_{2}\right)$ in $A B$ and $A C$ stacking. The unit is eV

\begin{tabular}{lllllll}
\hline & $\mathrm{ABA}\left(\mathrm{B}=\mathrm{MoSe}_{2}\right)$ & $\mathrm{ACA}\left(\mathrm{C}=\mathrm{MoSe}_{2}\right)$ & $\mathrm{ABA}\left(\mathrm{B}=\mathrm{WS}_{2}\right)$ & $\mathrm{ACA}\left(\mathrm{C}=\mathrm{WS}_{2}\right)$ & $\mathrm{ABA}_{\left(\mathrm{B}=\mathrm{WSe}_{2}\right)} \mathrm{ACA}_{\left(\mathrm{C}=\mathrm{WSe}_{2}\right)}$ \\
\hline$E_{\mathrm{g}}(\mathrm{PBE})$ & 0.70 & 0.75 & $1.05(I-k)$ & $1.08(\Gamma-k)$ & 0.39 & 0.42 \\
$E_{\mathrm{g}}(\mathrm{HSE})$ & 0.97 & 1.02 & $1.47(\Gamma-k)$ & $1.49(\Gamma-k)$ & 0.61 & 0.64 \\
$E_{\mathrm{g}}(\mathrm{PBE}) \_\mathrm{SL}$ & $0.62(\Gamma-k)$ & $0.59(\Gamma-k)$ & $0.95(\Gamma-k)$ & $0.93(\Gamma-k)$ & 0.30 & 0.35 \\
$E_{\mathrm{g}}(\mathrm{HSE}) \_\mathrm{SL}$ & 0.88 & 0.92 & $1.35(\Gamma-k)$ & $1.33(\Gamma-k)$ & 0.50 & 0.54
\end{tabular}




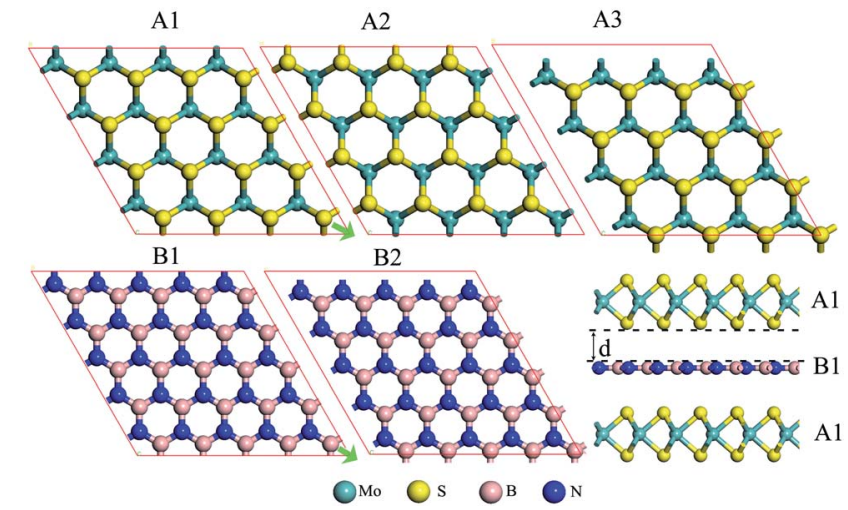

Fig. 1 Top view of a $\mathrm{MoS}_{2}$ monolayer in three different supercells (marked by the red parallelogram) and a BN monolayer in two different supercells. A3 (B2) can be viewed as a displacement of $A 1$ (B1) in the green-arrow direction shown in $A 1$ (B1). Superimposing the ABA supercells allows us to build different $\mathrm{MOS}_{2} / \mathrm{BN} / \mathrm{MOS}_{2}$ trilayers. An example of A1B1A1 trilayer is shown in the lower right panel.

monolayer, $E_{\mathrm{BN}}$ is the total energy of a $\mathrm{BN}$ monolayer, and $E_{\mathrm{MoS}_{2} / \mathrm{BN} / \mathrm{Mos}_{2}}$ is the total energy of a $\mathrm{MoS}_{2} / \mathrm{BN} / \mathrm{MoS}_{2}$ trilayer. As listed in Table 1, the computed binding energies of the $\mathrm{MoS}_{2} /$ $\mathrm{BN} / \mathrm{MoS}_{2}$ heterostructure with A1B1A1, A1B2A1, A1B1A2, and A1B1A3 stacking orders are $0.36 \mathrm{eV}, 0.36 \mathrm{eV}, 0.37 \mathrm{eV}$, and 0.36 $\mathrm{eV}$, respectively, reflecting the weak vdW interaction between the $\mathrm{MoS}_{2}$ layer and BN layer. Taking the A1B1A1 stacking as an example, its electronic structure is shown in Fig. 2c. Clearly, the trilayer retains the direct-gap character of the $\mathrm{MoS}_{2}$ monolayer. The computed bandgap is $1.58 \mathrm{eV}$, and both the conduction band minimum (CBM) and valence band maximum (VBM) are located at the $K$ points, both contributed by the $\mathrm{MoS}_{2}$ layers. Like the PBE calculation, the HSE06 calculation also suggests a direct-gap character but the bandgap increases to $2.00 \mathrm{eV}$ (ESI Fig. S1 $\dagger$ ). Overall, the intercalated BN layer has little effect on the band edge of $\mathrm{MoS}_{2}$ layers. To further confirm this conclusion, we remove the BN layer but leave the two $\mathrm{MoS}_{2}$ layers fixed at the original locations of the trilayer. As shown in Fig. 2b,

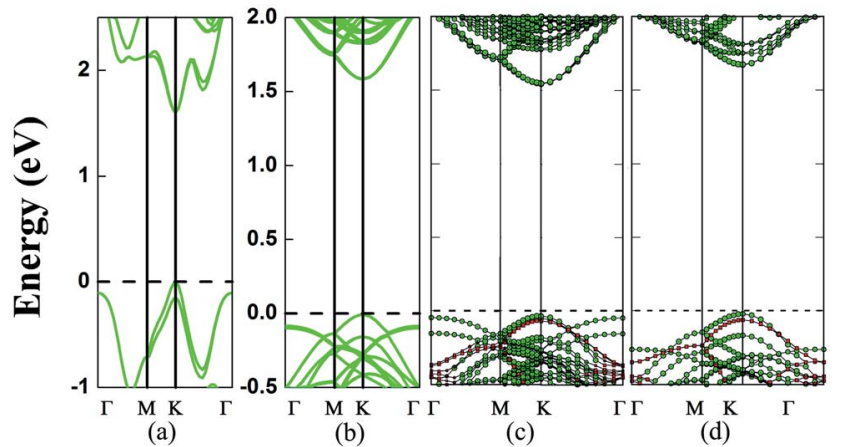

Fig. 2 Computed electronic band structures (PBE) of (a) monolayer $\mathrm{MoS}_{2}$; (b) $\mathrm{MoS}_{2} /$ vacuum layer/MoS 2 by removing the BN layer from the $\mathrm{MoS}_{2} / \mathrm{BN} / \mathrm{MoS}_{2}$ trilayer counterpart (in (c)) but with the fixed vertical location of the two $\mathrm{MoS}_{2}$ layers; (c) $\mathrm{MoS}_{2} / \mathrm{BN} / \mathrm{MoS}_{2}$ with the A1B1A1 stacking; and (d) a 3D superlattice of $\mathrm{MoS}_{2} / B N$ with the $A B$ stacking. The green lines represent $M_{2} S_{2}$ layers while the red lines represent BN monolayer. again, the computed band structure shows a direct-gap character with the bandgap being $1.59 \mathrm{eV}$, very close to that of the $\mathrm{MoS}_{2} / \mathrm{BN} / \mathrm{MoS}_{2}$ trilayer.

We also compute the effective mass at the $K$ point corresponding to the $\mathrm{MoS}_{2}$ monolayer and A1B1A1 trilayer, respectively. The directional dependence of the effective mass at the $K$ point is small. For the $\mathrm{MoS}_{2}$ monolayer, $m_{\mathrm{e}}^{*}=0.44 m_{0}$ for the electron at CBM and $m_{\mathrm{h}}^{*}=0.55 m_{0}$ for the hole at VBM are in agreement with the previous studies. ${ }^{4,44}$ For A1B1A1, $m_{\mathrm{e}}^{*}=$ $0.44 m_{0}$ and $m_{\mathrm{h}}^{*}=0.59 m_{0}$, similar to those of the monolayer, which indicates that the carrier mobility of the monolayer is also retained by the trilayer.

To confirm that the BN monolayer is an ideal sheet to decouple the interlayer coupling of the $\mathrm{MoS}_{2}$ bilayer, we also compute electronic structures of the 3D superlattice of hybrid $\mathrm{BN} / \mathrm{MoS}_{2}$ layers. A superlattice with two different stacking orders (A1B1 and A1B2) of $\mathrm{MoS}_{2}$ and BN layers is considered and our calculations show that the two stacking orders give nearly the same results. For both stacking orders, the optimized cell parameters are $a=b=12.62 \AA$ and $c=9.86 \AA$. As shown in Fig. 2d, the superlattice exhibits a direct gap of $1.69 \mathrm{eV}$, and both the VBM and CBM are located at the $K$ point and both are contributed by $\mathrm{MoS}_{2}$ layers as in the case of the $\mathrm{MoS}_{2} / \mathrm{BN} / \mathrm{MoS}_{2}$ trilayer system. The slightly enhanced bandgap compared to the trilayer system is largely due to a slight reduction of the cell parameters $a$ and $b$. In summary, results of both the vdW trilayer and superlattice show that the alternatively stacked BN and $\mathrm{MoS}_{2}$ monolayers can retain the direct-gap character of the $\mathrm{MoS}_{2}$ monolayer. In other words, the BN monolayer is an effective divider to decouple the interlayer coupling for $\mathrm{MoS}_{2}$.

\section{Intercalation of a $\mathrm{MoS}_{2}$ bilayer by $\mathrm{MoSe}_{2}, \mathrm{WS}_{2}$ or $\mathrm{WSe}_{2}$ monolayer}

Previous experimental and theoretical studies demonstrate that the $\mathrm{MoS}_{2}$ bilayer is a semiconductor with an indirect bandgap. ${ }^{27,29,45}$ Recent theoretical studies of TMDC heterobilayers also show that the interlayer interaction due to heterointerfaces (e.g., $\mathrm{S} / \mathrm{Se}$ ) can notably affect the electronic properties. Thus, it is interesting to study the extent to which the intercalation of a heterogeneous TMDC monolayer between two $\mathrm{MoS}_{2}$ bilayers affects the electronic properties.

Previous theoretical studies show that the $\mathrm{C} 7$ and $\mathrm{T}$ stacking patterns give the lowest energy for many heterobilayers, ${ }^{27,29}$ and the electronic structure is more or less the same with different stacking orders. Here, we adopt two different stacking orders for the trilayers (see Fig. 3), namely, the ABA and ACA. For the ABA trilayer, the interface $\mathrm{AB}$ is in $\mathrm{C} 7$ stacking, while for the $\mathrm{ACA}$ trilayer, the interface $\mathrm{AC}$ is in $\mathrm{T}$ stacking. Again, we find that the two stacking orders give rise to nearly the same electronic properties.

The polarization within the $\mathrm{S} / \mathrm{Se}$ interfaces is responsible for the direct-gap character of heterobilayers in previous studies. ${ }^{26,27}$ The $\mathrm{MoS}_{2} / \mathrm{MoSe}_{2} / \mathrm{MoS}_{2}$ trilayer entails two S/Se interfaces. In view of the $\mathrm{MoS}_{2}$ bilayer possessing an indirect bandgap, the intercalation of the $\mathrm{MoSe}_{2}$ monolayer induces an indirect to direct transition. As shown in Fig. 4a, the $\mathrm{MoS}_{2} /$ 
(a)

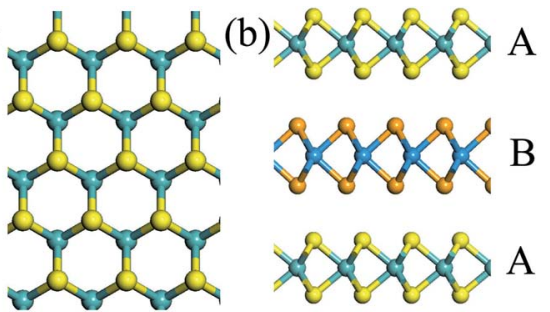

(c)

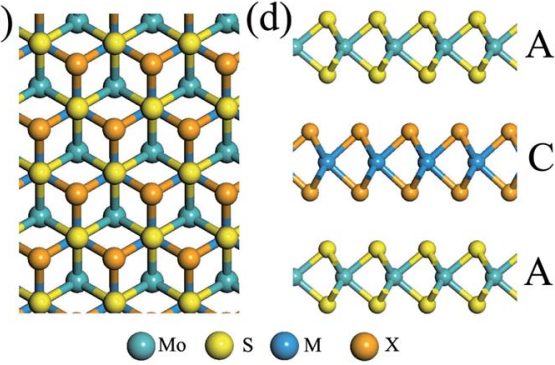

Fig. 3 Top and side views of $\operatorname{MoS}_{2} / M_{2} / M_{O} S_{2}(M=M o, W ; X=S, S e)$ trilayers with ( $a$ and $b$ ) ABA stacking with a $C 7$ interface and (c and d) ACA stacking with a $T$ interface, respectively.

$\mathrm{MoSe}_{2} / \mathrm{MoS}_{2}$ trilayer exhibits a direct bandgap of $0.69 \mathrm{eV}$. The VBM is located at the $K$ point and is mainly contributed by the $\mathrm{MoSe}_{2}$ layer, while the CBM is also located at the $K$ point and is mainly contributed by $\mathrm{MoS}_{2}$ layers. It is desirable that CBM and VBM are contributed from two different TMDC monolayers, particularly for the electron-hole separation. ESI (Fig. S2 $\dagger$ ) shows a more accurate HSE06 computation of band structures of the trilayers. The computed bandgap is $0.97 \mathrm{eV}$, wider than that from PBE computation. However, the electronic structures and the VBM and CBM are similar to those based on PBE computation.

Because of the lack of the $\mathrm{S} / \mathrm{Se}$ interfaces, as shown in Fig. $4 \mathrm{~b}$, the $\mathrm{MoS}_{2} / \mathrm{WS}_{2} / \mathrm{MoS}_{2}$ trilayer still exhibits an indirect gap of 1.05 $\mathrm{eV}$. The VBM is located at the $T$ point and is mainly contributed by the $\mathrm{WS}_{2}$ monolayer, while the CBM is located at the $K$ point and is mainly contributed by the two $\mathrm{MoS}_{2}$ layers. Computed band structures based on the HSE06 functional is shown in ESI Fig. S2. $\dagger$ Again, the trilayer still exhibits the indirect-gap character but the bandgap increases to $1.47 \mathrm{eV}$.

The $\mathrm{MoS}_{2} / \mathrm{WSe}_{2} / \mathrm{MoS}_{2}$ trilayer still exhibits a direct gap of $0.39 \mathrm{eV}$ due to the presence of the two Se/S interfaces (see Fig. 4c). The VBM at the $K$ point is mainly contributed by the $\mathrm{WSe}_{2}$ layer, while the CBM at the $K$ point is mainly contributed by $\mathrm{MoS}_{2}$ layers. Again, as shown in ESI Fig. S2, $\uparrow$ HSE06 calculation confirms the main character of electronic properties. To further analyze the effect of the polarization within the Se/S interfaces, charge transfer between neighboring layers is computed (see ESI Table S1 $\dagger$ ). For $\mathrm{MoS}_{2} / \mathrm{MoSe}_{2} / \mathrm{MoS}_{2}$ and $\mathrm{MoS}_{2} /$ $\mathrm{WSe}_{2} / \mathrm{MoS}_{2}$ trilayers, a $0.02 \mathrm{e}$ per unit cell is transferred from the $\mathrm{MoSe}_{2}$ (or $\mathrm{WSe}_{2}$ ) layer to the $\mathrm{MoS}_{2}$ layer. In contrast, for the $\mathrm{MoS}_{2} / \mathrm{WS}_{2} / \mathrm{MoS}_{2}$ trilayer with S/S interfaces, the charge transfer between two neighboring layers is nearly zero. This result further demonstrates that the interfacial polarization has an important effect on the electronic properties of the trilayer heterostructures.

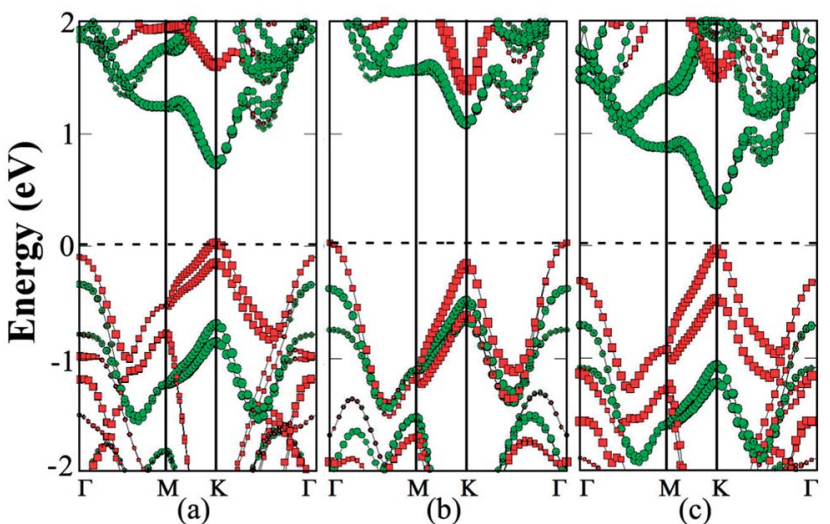

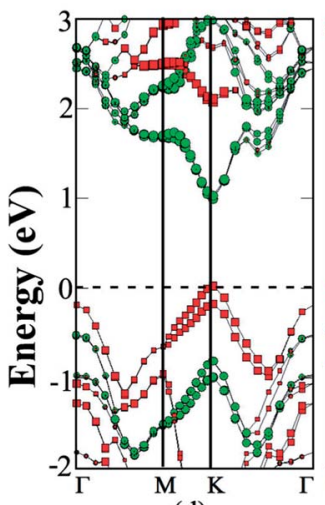

(d)

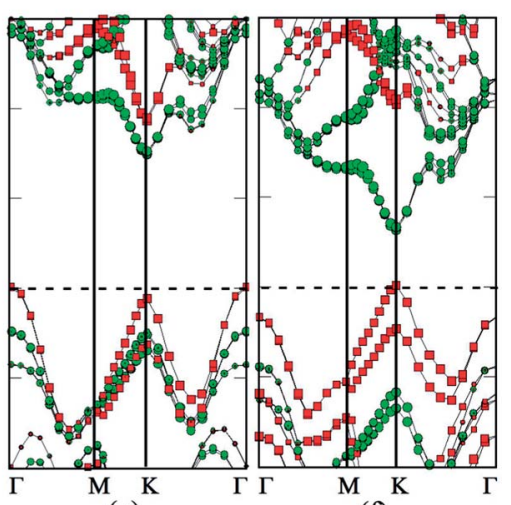

(e)

(f)
Fig. 4 Computed band structures (PBE) of (a) $\mathrm{MoS}_{2} / \mathrm{MoSe}_{2} / \mathrm{MoS}_{2}$, (b) $\mathrm{MoS}_{2} / \mathrm{WS}_{2} / \mathrm{MoS}_{2}$, and (c) $\mathrm{MoS}_{2} / \mathrm{WSe}_{2} / \mathrm{MoS}_{2}$ trilayer with ABA stacking, respectively. Computed band structures (PBE) of (d) $\mathrm{MoS}_{2} / \mathrm{MoSe}_{2}$ superlattice, (e) $\mathrm{MoS}_{2} / \mathrm{WS}_{2}$ superlattice, and (f) $\mathrm{MoS}_{2} / \mathrm{WSe}_{2}$ superlattice with the $A B$ stacking, respectively. The green lines mark contribution from $\mathrm{MoS}_{2}$ layers while the red lines mark contribution from the $\mathrm{MoSe}_{2}, \mathrm{WS}_{2}$ or $\mathrm{WSe}_{2}$ layer.

Lastly, we consider a 3D superlattice made of hybrid $\mathrm{MoS}_{2}$ monolayers and other monolayers. As shown in Fig. 4, for each superlattice, two stacking orders including $\mathrm{AB}$ with a $\mathrm{C} 7$ interface and $\mathrm{AC}$ with a $\mathrm{T}$ interface are investigated. The binding energies and cell parameters for the $\mathrm{AB}$ and $\mathrm{AC}$ stacking are close to one another in all the configurations (see ESI Table $\mathrm{S} 1 \dagger$ ). For the $\mathrm{MoS}_{2} / \mathrm{MoSe}_{2}$ superlattice, PBE calculations suggest that its bandgap is still indirect, with a value of 0.62 and $0.59 \mathrm{eV}$ respectively, for the $\mathrm{AB}$ and $\mathrm{AC}$ stacking. The bandgap is about $0.1 \mathrm{eV}$ less than that of the corresponding trilayer. The CBM is still located at the $K$ point and contributed mainly by the $\mathrm{MoS}_{2}$ layers (Fig. 4d), while the VBM energy at the $I$ and $K$ point differs only by $10 \mathrm{meV}$, and is mainly contributed by the $\mathrm{MoSe}_{2}$ layers. On the other hand, the HSE06 calculations suggest that the $\mathrm{MoS}_{2} / \mathrm{MoSe}_{2}$ superlattice is a direct-gap semiconductor with a value of 0.88 and $0.92 \mathrm{eV}$, respectively, for the $\mathrm{AB}$ and $\mathrm{AC}$ stacking. Here, the VBM energy in the $K$ point is $77 \mathrm{meV}$ lower than the $T$ point (ESI Fig. S2 $\uparrow$ ). For the $\mathrm{MoS}_{2} / \mathrm{WS}_{2}$ superlattice, both PBE and HSE06 calculations suggest that it is an indirectgap semiconductor (Fig. 4e) with a bandgap value 0.95 and 1.35 $\mathrm{eV}$, respectively, for the AB stacking. Finally, both PBE and HSE06 calculations suggest that the $\mathrm{MoS}_{2} / \mathrm{WSe}_{2}$ superlattice is a direct-gap semiconductor with a value of 0.3 and $0.5 \mathrm{eV}$ for the 
$\mathrm{AB}$ stacking, and 0.35 and $0.54 \mathrm{eV}$ for the AC stacking. Both bandgaps are smaller than those of the corresponding trilayers. Again, the bandgap reduction is mainly due to a slightly enlarged cell parameter (see ESI Table S1†).

\section{Conclusions}

In conclusion, our first-principles calculations show that the BN monolayer is a highly effective single sheet to decouple the interlayer vdW interaction of the $\mathrm{MoS}_{2}$ bilayer. The resulting vdW trilayer heterostructure can recover the electronic structures of a single $\mathrm{MoS}_{2}$ monolayer, particularly its direct-gap character. Further study of the 3D $\mathrm{MoS}_{2} / \mathrm{BN}$ superlattices confirms the effectiveness of the BN monolayer for decoupling the interlayer interaction. Expectedly, this conclusion has implications to $\mathrm{MoS}_{2}$ based heterostructures as well as to other TMDC-based vdW heterostructures. We have also investigated intercalation of a TMDC $\mathrm{MoSe}_{2}$ or $\mathrm{WSe}_{2}$ sheet between two $\mathrm{MoS}_{2}$ sheets and found that the resulting vdW trilayer undergoes an indirect-gap to direct-gap transition due to the newly formed heterogeneous $\mathrm{S} / \mathrm{Se}$ interfaces. In contrast, the $\mathrm{MoS}_{2} / \mathrm{WS}_{2} / \mathrm{MoS}_{2}$ vdW trilayer still retains the indirect-gap character of the $\mathrm{MoS}_{2}$ bilayer due to the lack of the heterogeneous S/Se interfaces. Again, the 3D superlattice of the $\mathrm{MoS}_{2}$ / TMDC heterostructures also exhibits similar electronic band characters to the $\mathrm{MoS}_{2} / \mathrm{TMDC} / \mathrm{MoS}_{2}$ trilayer, albeit a slight decrease of the bandgap than that of their trilayer counterparts. In view of recent successful fabrication of vdW heterostructures by stacking a graphene sheet on top of $\mathrm{MoS}_{2}$ sheets or vice versa,$^{19}$ the vdW trilayers and superlattices investigated in this study together with their novel properties may be tested in the laboratory in the near future.

\section{Acknowledgements}

XCZ is grateful for valuable discussions with Professors Ali Adibi, Eric Vogel, Joshua Robinson, and Ali Eftekhar. The USTC group is supported by the National Basic Research Programs of China (nos 2011CB921400 and 2012CB 922001), NSFC (grant nos 21121003, 11004180, and 51172223), and One Hundred Person Project of CAS, Strategic Priority Research Program of CAS (XDB01020300). The UNL group is supported by ARL (grant no. W911NF1020099), NSF (grant no. DMR-0820521), UNL Nebraska Center for Energy Sciences Research, University of Nebraska Holland Computing Center, and a grant from USTC for (1000plan) Qianren-B summer research.

\section{References}

1 B. Radisavljevic, A. Radenovic, J. Brivio, V. Giacometti and A. Kis, Nat. Nanotechnol., 2011, 6, 147-150.

2 H. Wang, L. L. Yu, Y. H. Lee, Y. M. Shi, A. Hsu, M. L. Chin, L. J. Li, M. Dubey, J. Kong and T. Palacios, Nano Lett., 2012, 12, 4674-4680.

3 B. Radisavljevic, M. B. Whitwick and A. Kis, ACS Nano, 2011, 5, 9934-9938.
4 Y. Yoon, K. Ganapathi and S. Salahuddin, Nano Lett., 2011, 11, 3768-3773.

5 K. F. Mak, C. Lee, J. Hone, J. Shan and T. F. Heinz, Phys. Rev. Lett., 2010, 105, 136805.

6 K. F. Mak, K. He, C. Lee, G. H. Lee, J. Hone, T. F. Heinz and J. Shan, Nat. Mater., 2012, 12, 207-211.

7 D. Xiao, G.-B. Liu, W. Feng, X. Xu and W. Yao, Phys. Rev. Lett., 2012, 108, 196802.

8 A. Splendiani, L. Sun, Y. Zhang, T. Li, J. Kim, C.-Y. Chim, G. Galli and F. Wang, Nano Lett., 2010, 10, 1271-1275.

9 D. Voiry, M. Salehi, R. Silva, T. Fujita, M. Chen, T. Asefa, V. B. Shenoy, G. Eda and M. Chhowalla, Nano Lett., 2013, 13, 6222-6227.

10 H. L. Zhuang and R. G. Hennig, Chem. Mater., 2013, 25, 3232-3238.

11 S. Bertolazzi, J. Brivio and A. Kis, ACS Nano, 2011, 5, 97039709.

12 J. Pu, Y. Yomogida, K.-K. Liu, L.-J. Li, Y. Iwasa and T. Takenobu, Nano Lett., 2012, 12, 4013-4017.

13 Y. Li, Z. Zhou, S. Zhang and Z. Chen, J. Am. Chem. Soc., 2008, 130, 16739-16744.

14 K. F. Mak, C. Lee, J. Hone, J. Shan and T. F. Heinz, Phys. Rev. Lett., 2010, 105, 136805.

15 H. J. Conley, B. Wang, J. I. Ziegler, R. F. Haglund, S. T. Pantelides and K. I. Bolotin, Nano Lett., 2013, 13, 3626-3630.

16 E. S. Kadantsev and P. Hawrylak, Solid State Commun., 2012, 152, 909-913.

17 A. Geim and I. Grigorieva, Nature, 2013, 499, 419-425.

18 L. Ponomarenko, A. Geim, A. Zhukov, R. Jalil, S. Morozov, K. N. I. Grigorieva, E. Hill, V. Cheianov, V. Fal'ko and K. Watanabe, Nat. Phys., 2011, 7, 958-961.

19 L. Britnell, R. Gorbachev, R. Jalil, B. Belle, F. Schedin, A. Mishchenko, T. Georgiou, M. Katsnelson, L. Eaves and S. Morozov, Science, 2012, 335, 947-950.

20 S. Haigh, A. Gholinia, R. Jalil, S. Romani, L. Britnell, D. Elias, K. Novoselov, L. Ponomarenko, A. Geim and R. Gorbachev, Nat. Mater., 2012, 11, 764-767.

21 S. Bertolazzi, D. Krasnozhon and A. Kis, ACS Nano, 2013, 7, 3246-3252.

22 T. Georgiou, R. Jalil, B. D. Belle, L. Britnell, R. V. Gorbachev, S. V. Morozov, Y.-J. Kim, A. Gholinia, S. J. Haigh and O. Makarovsky, Nat. Nanotechnol., 2013, 8, 100-103.

23 W. J. Yu, Z. Li, H. Zhou, Y. Chen, Y. Wang, Y. Huang and X. Duan, Nat. Mater., 2012, 12, 246-252.

24 A. Anto Jeffery, C. Nethravathi and M. Rajamathi, J. Phys. Chem. C, 2013, 118, 1386-1396.

25 H.-P. Komsa and A. V. Krasheninnikov, Phys. Rev. B: Condens. Matter Mater. Phys., 2013, 88, 085318.

26 H. Terrones, F. López-Urías and M. Terrones, Sci. Rep., 2013, 3, 1549.

27 (a) N. Lu, H. Guo, L. Li, J. Dai, L. Wang, W.-N. Mei, X. Wu and X. C. Zeng, Nanoscale, 2014, 6, 2879-2886; (b) H. Guo, N. Lu, L. Wang, X. Wu and X. C. Zeng, J. Phys. Chem. C, 2014, 118, DOI: $10.1021 /$ jp501734s.

28 (a) Y. Ma, Y. Dai, M. Guo, C. Niu and B. Huang, Nanoscale, 2011, 3, 3883-3887; (b) J. Kang, J. Li, S-S. Li, J.-B. Xia and 
L.-W. Wang, Nano Lett., 2013, 13, 5485-5490; (c) J. Kang, S. Tongay, J. Zhou, J. Li, and J. Wu, Appl. Phys. Lett., 2013, 102, 012111.

29 K. Kośmider and J. Fernández-Rossier, Phys. Rev. B: Condens. Matter Mater. Phys., 2013, 87, 075451.

30 C. Dean, A. Young, I. Meric, C. Lee, L. Wang, S. Sorgenfrei, K. Watanabe, T. Taniguchi, P. Kim and K. Shepard, Nat. Nanotechnol., 2010, 5, 722-726.

31 K. Novoselov and A. C. Neto, Phys. Scr., 2012, 2012, 014006.

32 G. Kresse and J. Furthmüller, Phys. Rev. B: Condens. Matter Mater. Phys., 1996, 54, 11169.

33 G. Kresse and J. Furthmüller, Comput. Mater. Sci., 1996, 6, 15-50.

34 G. Kresse and J. Hafner, Phys. Rev. B: Condens. Matter Mater. Phys., 1993, 47, 558.

35 G. Kresse and D. Joubert, Phys. Rev. B: Condens. Matter Mater. Phys., 1999, 59, 1758.

36 J. P. Perdew, K. Burke and M. Ernzerhof, Phys. Rev. Lett., 1996, 77, 3865 .
37 J. Klimeš, D. R. Bowler and A. Michaelides, J. Phys.: Condens. Matter, 2010, 22, 022201.

38 J. Klimeš, D. R. Bowler and A. Michaelides, Phys. Rev. B: Condens. Matter Mater. Phys., 2011, 83, 195131.

39 C. Ataca, H. Sahin and S. Ciraci, J. Phys. Chem. C, 2012, 116, 8983-8999.

40 H. L. Zhuang and R. G. Hennig, J. Phys. Chem. C, 2013, 117, 20440-20445.

41 E. Sanville, S. D. Kenny, R. Smith and G. Henkelman, J. Comput. Chem., 2007, 28, 899-908.

42 J. Heyd, G. E. Scuseria and M. Ernzerhof, J. Chem. Phys., 2006, 124, 219906.

43 Y.-S. Kim, K. Hummer and G. Kresse, Phys. Rev. B: Condens. Matter Mater. Phys., 2009, 80, 035203.

44 A. Sengupta, R. K. Ghosh and S. Mahapatra, IEEE Trans. Electron Devices, 2013, 60, 2782-2787.

45 Y. Y. Hui, X. Liu, W. Jie, N. Y. Chan, J. Hao, Y.-T. Hsu, L.-J. Li, W. Guo and S. P. Lau, ACS Nano, 2013, 7, 71267131. 